\title{
Optimization of University Campus Takeout Joint Distribution Route Based on Clustering Tabu Search Algorithm
}

\author{
Shuaisha Sun \\ Beijing jiaotong university, Beijing, China \\ sshuaisha@163.com
}

Keywords: Campus takeout, Joint distribution, Clustering algorithm, Tabu search algorithm, Route optimization

\begin{abstract}
With the rapid development of the Internet, takeout in campus also conform to the general trend of the times. However, because of the low efficiency and not timely delivery, the campus takeout distribution is facing great challenges. In this paper, according to the features of campus takeout. The mathematical model of joint distribution system optimization is established in campus with the target of reducing the average delivery time. Taking Shanxi University takeout distribution as an example, clustering algorithm is used to partition., then a tabu search is used to plan the route for a single area. With the optimization of the distribution system, the whole logistics rate , customer satisfaction and business interests can be improved.
\end{abstract}

\section{Introduction}

According to relevant research, more and more users order meals online. But the problem of food and beverage distribution is faced with many problems. Logistics distribution system is relatively backward, hindering the development of catering trade. Now, people have a higher demand for logistics services. So it is very important to solve the problem of distribution. But because of the number of customers and the influence of the external environment and other necessary factors, the distribution of the University takeout is becoming more and more complex.

Foreign research path optimization problem for many years. Dantzig and Ramser are the earliest idea of line optimization. In 1959, Dantzig and Ramser first proposed the problem of route optimization research [1]. Send the least quantity of vehicles from the distribution center, choose the right line, and deliver the goods to the customers in the shortest and most efficient way [2].Miller proposed a new algorithm - scanning algorithm to group demand points through ray rotation and vehicle volume constraints [3]. Glover proposed a tabu search algorithm, which is then partially searched and then gradually extended to achieve global optimization [4]. The ant colony algorithm proposed by M.Dorigo does not depend on the initial path. It is an algorithm of optimal path search through positive feedback and self-organization. To a certain extent, it can solve difficult combinatorial optimization problems [5]. Wei Ming et al. used a tabu search algorithm to solve a line optimization problem with time windows and capacity constraints, and could obtain a better solution faster [6]. Zhong Shiquan studied various intelligent optimization methods and compared their various performance in various vehicle scheduling problems [7].

In this paper, we need to optimize the distribution routes of takeout in universities. Optimizing delivery routes can improve the timeliness of employees, reduce labor costs, quickly and accurately fulfill customer requirements, and bring benefits to stores.

\section{Problem and Method}

\subsection{Problem}

The university campus takeout occupies an important position in the takeout, accounting for $26.8 \%$ of the third platforms. Most of the distribution is the independent distribution of the business. But there's 
always a problem of efficiency, Because of the long meal time, the complaint caused by the college students has greatly reduced the level of service. The main problems are as follows:

First: the unreasonable division of the distribution area makes the invalid journey too much. In the delivery process, a long distance order is delivered to the same distributor at the same time, resulting in too many distribution routes and reducing the timeliness of delivery.

Second, the unreasonable planning of the line makes the repetition rate of the road too high. In the process of University takeout distribution, due to the "repeat" distribution caused by the different distribution members who take the same area for takeout, the distribution efficiency also results in the waste of manpower. Moreover, the dispatcher will not make route planning according to reason, but make the distribution route according to his personal habits, which will cause waste and duplication of delivery time and distance.

\subsection{Route optimization algorithm}

In clustering algorithm, K-means algorithm has high efficiency in dealing with large-scale problems, and is easy to describe and implement. It is fast and simple. So clustering algorithm in this paper uses $\mathrm{K}$-means to solve the grouping problem in line optimization.

\subsubsection{K-means clustering algorithm}

The K-means algorithm, which was proposed by MacQeen in 1967, is the most widely used clustering algorithm. The first step of the solution has a great influence on the later stage of the algorithm.

Method: Based on the average value of the objects in the clustering.

Algorithm steps:

a) select the initial value of the $\mathrm{K}$ cluster center.

b) calculate the distance between each node to the k center and classify it into the nearest class.

c) the new cluster center values in each class are calculated by means of the average method.

d) cycle second, third step, when the central value of K clustering no longer changes, then the loop ends.

\subsubsection{Tabu search algorithm}

The idea of tabu search is an extension of local domain search, and it is a global optimization algorithm. It ensures global optimization by Tabu tabu and tabu criteria and contempt criteria.

Algorithm steps :

a) according to the actual situation, the parameters are set, the initial solution is randomly obtained, and the tabu table is empty.

b) do you know whether the end of the algorithm is satisfied? The output is satisfied, otherwise it will continue to run.

c) make use of the neighborhood function of the current solution to produce the neighborhood solution, and then determine some candidate solutions from it.

d) is it satisfied to judge the scorn criterion for the candidate solution? If it is established, it will replace the $\mathrm{X}$ with the best state $\mathrm{y}$ to become the new current solution, that is, $\mathrm{x}=\mathrm{y}$, and use taboo objects corresponding to y to replace taboo objects which first enter taboo tables. At the same time, replace the "best so far" state with y, then turn to step 6. Otherwise, continue the following steps.

e) judge the tabu attributes of corresponding objects, select the best state corresponding to the non-taboo objects in the candidate solution set as the new current solution, and use the corresponding taboo object to replace taboo object elements which first entered tabu list.

f) turn step 2 .

\section{Construction and Application of the Model}

\subsection{Construction of optimization model}

\subsubsection{The definition of a variable}

Distribution car $\mathrm{K}$ vehicles, each vehicle transport volume of $\mathrm{Qk}(\mathrm{k}=1,2 \ldots \mathrm{K})$, the car's driving distance is $\mathrm{Dk}(\mathrm{k}=1,2 \ldots, \mathrm{K})$. The distribution of the apartment has $\mathrm{L}$, distribution volume for each 
apartment is Qi; The distance between the apartment $\mathrm{i}$ and the apartment $\mathrm{j}$ is Dij, the distribution center 0 , the number of each car vehicle distribution apartment is $n$, car route is $R$.

\subsubsection{Mathematical model}

$$
\begin{aligned}
& \operatorname{minZ}=\sum_{\mathrm{k}=1}^{\mathrm{k}}\left[\sum_{\mathrm{i}=1}^{\mathrm{n}_{\mathrm{k}}} \mathrm{d}_{\mathrm{r}_{\mathrm{k}(\mathrm{i}-1)} \mathrm{r}_{\mathrm{ki}}}+\mathrm{d}_{\mathrm{r}_{\mathrm{kn}} \mathrm{r}_{\mathrm{k} 0}} \times \operatorname{sign}\left(\mathrm{n}_{\mathrm{k}}\right)\right] \\
& \operatorname{minZ}=\sum_{\mathrm{k}=1}^{\mathrm{k}} \operatorname{sign}\left(\mathrm{n}_{\mathrm{k}}\right) \\
& \sum_{\mathrm{i}=1}^{\mathrm{n}_{\mathrm{k}}} \mathrm{q}_{\mathrm{r}_{\mathrm{ki}}} \leq \mathrm{Q}_{\mathrm{k}} \\
& \sum_{\mathrm{i}=1}^{\mathrm{n}_{\mathrm{k}}} \mathrm{d}_{\mathrm{r}_{\mathrm{k}(\mathrm{i}-1)} \mathrm{r}_{\mathrm{ki}}}+\mathrm{d}_{\mathrm{r}_{\mathrm{kn}} \mathrm{n}_{\mathrm{k} \mathrm{k}} \mathrm{r}_{\mathrm{k}} \times \operatorname{sign}\left(\mathrm{n}_{\mathrm{k}}\right) \leq \mathrm{D}_{\mathrm{k}}} \\
& 0 \leq \mathrm{n}_{\mathrm{k}} \\
& \sum_{\mathrm{k}=1}^{\mathrm{k}} \mathrm{n}_{\mathrm{k}}=\mathrm{K} \\
& \mathrm{R}_{\mathrm{k}}=\left\{\mathrm{r}_{\mathrm{ki}} \mid \mathrm{r}_{\mathrm{ki}} \in\{1, \quad \ldots, \quad \mathrm{K}\}, \mathrm{i}=1,2 \ldots, \mathrm{n}_{\mathrm{k}}\right. \\
& \operatorname{Sign}\left(\mathrm{n}_{\mathrm{k}}\right)=\left\{\begin{array}{c}
1 \mathrm{n}_{\mathrm{k}} \geq 1 \\
0 \text { others }
\end{array}\right.
\end{aligned}
$$

The formula (1) indicates that the route of distribution is minimum. The formula (2) indicates that the number of vehicles required to be delivered is less. The formula (3) indicates that the vehicle volume is limited. The formula (4) indicates the limitation of the distribution route. The formula (5) indicates that the number of each distribution apartment is more than or equal to zero. The formula (6) indicates that all the apartments are served. The formula (7) represents the order of the route; The formula (8) indicates that when the distribution vehicle is delivered, the apartment is larger than 1, indicating that the car has been used, otherwise, it is not used.

\subsection{Line optimization simulation-K-means clustering algorithm}

\subsubsection{Select the initial cluster center}

Consider the needs of the students at Shanxi University, the intensity of the apartment and the full load of the car. In this paper, 4 initial cluster centers are determined.

Similarity measure: This article uses the Euclidean distance, that is, the relative distance between the dorm and the dorm.

$$
\begin{gathered}
\mathrm{K}(\mathrm{x})=\min \sum_{\mathrm{i}=1}^{\mathrm{k}}\left[\left(\mathrm{x}_{\mathrm{i}}-\overline{\mathrm{x}}\right)^{2}+\left(\mathrm{y}_{\mathrm{i}}-\overline{\mathrm{y}}\right)^{2}\right] \\
\text { Thereinto } \quad \overline{\mathrm{x}}=\sum_{\mathrm{i}=1}^{\mathrm{n}} \mathrm{x}_{\mathrm{i}} / \mathrm{n}, \quad \overline{\mathrm{y}}=\sum_{\mathrm{i}=1}^{\mathrm{n}} \mathrm{y}_{\mathrm{i}} / \mathrm{n} \\
\mathrm{K}, \quad \mathrm{i}=1,2, \quad \ldots, \mathrm{n}
\end{gathered}
$$

\subsubsection{Uses SPSS to run k-means algorithm}

Using SPSS to cluster the dormitory building of Shanxi University, 4 clusters are obtained. The results are as follows:

Table 1 Distribution area aggregation table

\begin{tabular}{lllll}
\hline \multicolumn{5}{c}{ gather } \\
\hline & 1 & 2 & 3 & 4 \\
\hline 01 & 16 & 83 & 72 & 31 \\
02 & 9 & 35 & 24 & 8 \\
\hline
\end{tabular}

\subsection{Line optimization-Tabu search algorithm}

Use matlab to run the algorithm. Run the program and get the following results. 
Table 2 distribution path planning table

\begin{tabular}{|l|l|l|l|}
\hline Cluster & Vehicle number & Distance $(\boldsymbol{m})$ & Route \\
\hline 1 & 3 & 7100 & $0-16-17-0-18-0-21-20-19-22-0$ \\
\hline 2 & 6 & 13000 & $0-1-2-0-3-4-0-5-6-0-7-8-0-9-12-0-10-11-0$ \\
\hline 3 & 4 & 4500 & $0-14-15-0-29-13-0-30-31-0-32-0$ \\
\hline 4 & 2 & 2200 & $0-23-24-25-26-0-27-28-0$ \\
\hline
\end{tabular}

Through the MATLAB operation tabu search algorithm, according to the demand of each building, as well as the full load of the distribution car, get the route planning of the upper table. A total of 15 cars will be needed, with a total distance of 26800 meters.

\section{Conclusions and Suggestions}

Based on the study of university campus takeaway delivery problems, based on the campus common distribution model, in order to avoid the distribution of different distribution staff in the same place, in order to reduce the delivery staff at the same time in the process of delivery, repeat route, proposed route optimization scheme. First, the K-means clustering algorithm is used to subregion the distribution area, so as to avoid the same route for several distribution personnel. Then the tabu search algorithm is used to plan the distribution line, so as to avoid repeat route for the distributor. At the same time, the number of distribution personnel is reduced by line optimization, and the interests of the merchants are improved.

For the optimization of the campus takeout line, the implementation of the offline entity shop requires;

First, to negotiate each business, let the merchant agree to adopt the common distribution pattern;

Second, build a campus takeaway distribution platform, gather business order information, so that the distributor can get his order information in time, and pick up and deliver goods.

Finally, the seamless connection between the university campus takeaway distribution system and the online order platform is achieved, so that the information of the order can be accurately delivered to the university campus takeaway distribution system for distribution providers.

\section{References}

[1] Dabbzubg G, Ramser I. The truck diskatching kroblem Management Science, 1959, 10(6) : $80-9$

[2] Kaolo Toth, Daniele Vigo.THE VEHICLE ROUTING KROBLEM,Society for Industrial and Akklied Mathematics khiladekhia.2002

[3] Gillett B E' Miller L R A Heuristic Algorithm for the Vehic le-Diskatch Kroblem. Okerations Research, 1974, 22(2)'. 340- 349.

[4] Glover F. Tabu Search-Kart I. ORSAIournal on Comkuting, 1989, 1(3) : 190- 206.

[5] Macro Dorigo, Thomas Stutzle., Zhang Jun, Hu Xiaomin, Luo Xuyao, et al. Ant colony optimization. Beijing: Tsinghua University press, 2007.

[6] Guo Yaohuang, Li Jun. Vehicle optimal scheduling. Chengdu: Chengdu University of Science and Technology press, 1994.

[7] Wei Ming, Gao Cheng $\mathrm{Hu}, \mathrm{Hu}$ Run Zhou. A vehicle routing problem with time windows and capacity constraints and its Tabu Search algorithm. Operations research and management, 2002, $11(3): 49-53$. 